\title{
QUELLE PLACE POUR LA PSYCHOLOGIE DANS LE POSITIVISME?
}

\author{
Annie PETIT
}

RÉSUMÉ : La "psychologie " n'est pas intégrée dans l'échelle des sciences du positivisme comtien. Comte juge même qu'elle est le refuge de la vieille "métaphysique ". Cependant, "les phénomènes affectifs, intellectuels et moraux " devant, eux aussi, être étudiés "positivement", Comte en appelle d'une part à la "biologie " où il élabore une " théorie cérébrale ", et d'autre part à la " sociologie " et à sa méthode historique. Puis il programme une nouvelle science, une "moraleanthropologie", où se retrouve une grande partie des questions qui relevaient de l'ex-psychologie.

On analyse aussi les discussions de Comte avec des proches plus ou moins disciplinés : John Stuart Mill ironise sévèrement contre la théorie cérébrale et réclame une place pour la "logique »; Émile Littré, disciple plus nuancé, accepte une "psychophysiologie», mais conteste maints développements et conséquences qu'envisageait Comte.

Lorsqu'il classe les sciences fondamentales, dans le Cours de philosophie positive, Auguste Comte n'accorde pas de place à la «psychologie ». Pire, il n'en parle que pour lui réserver des critiques sévères. Il lui refuse en fait tout statut de discipline scientifique. Mais alors où et comment traiter des phénomènes affectifs, intellectuels et moraux, ce que Comte prétend pourtant faire? De quelle(s) discipline(s) positive(s) doivent-ils relever?

On précisera d'abord les critiques comtiennes, car il s'agit de savoir quelle est au juste la psychologie expulsée. On montrera ensuite que ce qui relevait de la «psychologie » est écartelé et annexé, d'une part, par la biologie - plus particulièrement dans une «théorie cérébrale » inspirée par Gall - et, d'autre part, par ce que Comte dénomme alors « sociologie ». On rappellera combien et comment ces questions du statut de la «psychologie » et de ses relations avec la biologie et la sociologie ont été pour ou contre - le positivisme déterminantes : âprement débattues par les adversaires, mais aussi souvent par les différents adeptes. On évoquera Revue de synthèse : IV S. Nos 3-4, juillet-décembre 1994. 
donc la manière dont certains disciples indisciplinés ont redessiné le territoire et les frontières d'une psychologie positiviste' .

I. - PROCES ET NON-LIEU DE LA « PSYCHOLOGIE 》

DANS LA PHILOSOPHIE POSITTVE D'AUGUSTE COMTE

\section{1. - L'INFLATION DES CRITIQUes.}

1) Dès 1819 , les thèmes essentiels du procès sont installés :

«L'esprit de l'homme, considéré en lui-même, ne peut pas être un sujet d'observation, car chacun ne peut point, évidemment, l'observer dans autrui; et d'un autre côté, il ne peut pas non plus l'observer en lui-même. Et en effet, on observe les phénomènes avec son esprit; mais avec quoi observerait-on l'esprit lui-même, ses opérations, sa marche? On ne peut pas partager son esprit, c'est-à-dire son cerveau, en deux parties, dont l'une agit, tandis que l'autre la regarde faire, pour voir de quelle manière elle s'y prend; croire cela possible, c'est tomber dans la même erreur, c'est se faire la même illusion que lorsqu'on dit que nous voyons les objets parce que leurs images se peignent au fond de l'æxil. Mais avec quoi voyez-vous les images? repondent les physiologistes. Il vous faudrait un autre wil pour les regarder, si les impressions lumineuses agissaient comme images sur votre rétine. Il en est de même ici : vous voulez observer votre esprit, mais avec quoi le regarderez-vous? Il vous en faudrait un autre pour l'examiner. Il résulte de là que les prétendues observations faites sur l'esprit humain consideré en luimême et a priori sont de pures illusions; et qu'ainsi, tout ce qu'on appelle logique, métaphysique, idéologie, est une chimère et une rêverie, quand ce n'est point une absurdité ${ }^{2}$.

Le jeune polytechnicien ne croit qu'aux expériences dûment contrôlées. La critique est virulente : la soi-disant étude de l'esprit ou de l'âme n'est que « prétendues observations », " erreur », « illusions ", «chimères", "rêverie », " absurdité ». L'essentiel de l'argumentation critique porte sur la méthode : il n'y a pour Comte d'observations valables, et donc susceptibles d'offrir des inférences scientifiques, que s'il y a extériorité de l'observateur; toute introspection est invalidée. Par ailleurs, cette rapide fin de non-recevoir renvoie à la physiologie comme science-modèle, et oriente la réflexion vers l'étude des conditions des processus cérébraux. On aura remarqué au passage l'équivalence entre esprit et cerveau.

1. Pour les références aux ouvres principales et leurs abréviations, voir la Bibliographie, infro, p. $415-416$.

2. Corr., I, Iettre à Valat, 24 sept. 1819, p. $58-59$. 
2) Comte n'aborde cependant pas directement ces questions dans ses opuscules de jeunesse, qui suivent deux directions : scientifique et politique. En science, Comte vise à reconstruire la philosophie des mathématiques, voulant en systématiser la distribution des parties et fonder une pédagogie mieux ordonnée ${ }^{3}$. En politique, il s'agit de chercher comment arrêter l'enchaînement des désordres et des violences, de réorganiser la société avec répartition des rôles et des pouvoirs, ajustement des forces, et de trouver une véritable politique scientifique basée sur l'observation réfléchie du passé pour préparer l'avenir dans l'ordre et le progrès ${ }^{4}$. Dans ces deux directions, pourtant, Comte rencontre des questions de "psychologie » : dans la première, parce qu'il élargit ses réflexions à la logique, aux processus intellectuels et au fonctionnement de l'esprit humain; dans la seconde, la lecture de l'histoire le mène à des méditations de « psychologie collective » - réflexions sur la formation et l'efficacité des « opinions » et des «désirs", sur l'importance majeure de «l'opinion publique», sur la distribution des rôles entre " gouvernants » et " gouvernés », sur les affrontements des groupes et des classes, sur la part d'efficace humaine, celle des génies et grands hommes et celle des peuples et des masses ${ }^{5}$... Les deux programmes convergent en fait parce que Comte en vient vite à l'idée qu'un nouvel ordre social ne pourra s'établir que lorsque les esprits participeront tous d'une doctrine commune, partagée car scientifiquement assurée : la philosophie scientifique apparaît comme la voie de la philosophie socio-politique. Comte s'interroge aussi sur la nécessité de faire soutenir la politique scientifiquement raisonnée par d'autres forces collectives, seules capables d'y faire adhérer les masses : il faut trouver les moyens de créer " la persuasion vive et entraînante produite par les idées qui émeuvent les passions $" 6$.

Et lorsque, après sa rupture avec Saint-Simon, Comte se lance dans les Considérations qui décident de son propre parcours, il appuie aussi ses méditations et programmes politiques sur une sorte de psychologie générale de «l'homme » entrelaçant références au biologique et à l'historique?

3. Voir différents Essais sur la philosophie des mathématiques, de 1819, 1820, 1821, regroupés in E.J., p. 491-573.

4. La rencontre et la collaboration avec Saint-Simon (de fin 1817 à 1824) ont été sur ces points déterminantes. Mais Comte était sensibilisé aux questions politiques avant de rencontrer Saint-Simon : voir Henri GouHier, La Jeunesse d'Auguste Comte et la formation du positivisme, 3 t., Paris, Vrin, 1933, 1936, 1941, t. I surtout.

5. Voir les opuscules de jeunesse que Comte lui-même a jugé bon de republier en « Appendice ", à la fin du Système, pour bien montrer la continuité de ses préoccupations.

6. Plan, p. 104-105. Il faut aussi, dit Comte, « satisfaire le besoin moral d'exaltation propre à l'homme " (ibid.).

7. Comte plaide cependant pour la conception de l'originalité propre de la science sociale : s'il faut l'appuyer sur la physiologie, il ne saurait être question de l'y réduire (voir Plan, 
On n'en retiendra ici que deux thèmes : d'une part, l'idée que c'est l'affectivité qui guide et éveille les facultés cognitives de l'homme, d'abord " engourdies " - c'est la volonté de puissance qui entrâne la volonté de savoir $^{8}$; d'autre part, sur le plan social, Comte affirme, pour qu'une société se forme et se maintienne, la nécessité d'une "communion d'idées », d'un « système d'idées quelconque, capable de surmonter l'opposition des tendances individuelles, si prononcées à l'origine, et de les faire concourir en un ordre constant ${ }^{9}$, c'est-à-dire ce que nous appellerions une cohésion idéologique ${ }^{10}$.

Le Cours de philosophie positive est précisément entrepris pour établir au moins les conditions de l'accord intellectuel et spirituel d'où l'harmonie politique est espérée. Dans l'« Annonce » de son Cours, Comte précise ces objectifs, en soulignant d'ailleurs qu'il remplira ainsi le programme pour lequel "la prétendue méthode psychologique " s'est avérée impuissante ${ }^{11}$ et ces idées sont reprises dans les leçons d'ouverture ${ }^{12}$.

Ainsi, bien qu'il refuse d'admettre que la "psychologie " puisse être un domaine propre de positivité scientifique, on voit que Comte mêle étroitement, de fait, des considérations "psychologiques " aux questions abordées. Refoulée comme science, la «psychologie » est omniprésente dans

p. 124-129), et s'il affirme bien que " les fonctions intellectuelles et affectives relèvent de la physiologie, il invite à les distinguer des " autres fonctions organiques " (C.S.S., p. 148-149).

8. C.S.S., p. 137-143.

9. C.S.S., p. 159,143

10. Dans C.P.S., p. 184-189, 193-194, 211-215, Comte réclame aussi une doctrine et une discipline communes, et un bon " gouvernement de l'opinion $"$

11. Voir * Cours de philosophie positive, Annonce *, Revue encyclopédique, 9 sept. 1829, repr. in E.J., p. 573 : «Outre l'importance propre et directe d'une telle opération, comme dernier complément nécessaire de la grande régénération intellectuelle commencée par Bacon, par Descartes, et par Galilée, cette nouvelle série de travaux [...] présente pour les progrès généraux de l'esprit humain, quatre propriétés principales de la plus haute importance :$1^{\circ}$ En étudiant la marche effective de notre entendement dans ses travaux les plus étendus et les plus parfaits, elle peut seule manifester par expérience les lois que suivent dans leur accomplissement nos fonctions intellectuelles, et, par suite, déterminer la manière de procéder convenablement à la découverte de la vérité; double étude capitale, abandonnée jusqu'ici à la prétendue méthode psychologique, qui n'a rien produit et ne peut rien produire, comme étant radicalement nulle dans son principe. $-2^{\circ}$ Elle doit permettre la réorganisation de notre éducation générale $[\ldots]-3^{\circ}$ Cette étude spéciale des généralités scientifiques est même destinée à procurer aux sciences particulières de nouveaux moyens d'avancement $[\ldots]-4^{0}$ Enfin, le triomphe général et définitif de la philosophie positive [...] peut seul déterminer le retour des sociêtés actuelles à un état normal, la crise révolutionnaire de l'Europe moderne tenant surtout à l'anarchie intellectuelle. *

12. Voir, par ex., $C ., 1^{*} 1$., I, p. 38 : « Les idées bouleversent et gouvernent le monde, ou en d'autres termes, tout le mécanisme social repose finalement sur des opinions [...] Notre mal le plus grave consiste, en effet, dans cette profonde divergence qui existe maintenant entre tous les esprits relativement à toutes les maximes fondamentales dont la fixité est la première condition d'un véritable ordre social. Tant que les intelligences n'auront pas adhéré par un sentiment unanime à un certain nombre d'idées générales capables de fonder une doctrine sociale commune, on ne peut se dissimuler que l'état des nations restera de toute nécessité essentiellement révolutionnaire. » Voir aussi $C$., $1^{\text {re }}$ l., I, p. 35-36, $2^{\mathrm{E}}$ 1., p. 60-62. 
les interrogations comtiennes, elle investit ses méditations scientifiques et politiques, et, somme toute, les relie.

3) Mention particulière doit être faite, dans les ouvres de jeunesse de Comte, d'un texte de 1828 : Examen du traité de Broussais sur l'irritation et la folie. Il condense les diverses critiques de la « psychologie » et Comte se fait avocat d'une attitude scientifique nouvelle, véritable "révolution " dans la compréhension des "phénomènes intellectuels et affectifs ", dont Cabanis et Gall sont donnés pour les artisans :

"Tous les esprits vraiment au niveau de leur siècle [...] regardent l'étude des fonctions intellectuelles et affectives comme inséparablement liée à celle de tous les autres phénomènes physiologiques, et comme devant être poursuivie par les mêmes méthodes et dans le même esprit. Cependant quelques hommes [...] ont essayé depuis dix ans de transplanter parmi nous la métaphysique allemande, et de constituer sous le nom de psychologie une prétendue science entièrement indépendante de la physiologie, supérieure à elle, et à laquelle appartiendrait exclusivement l'étude des phénomènes spécialement appelés moraux. Quoique ces tentatives rétrogrades ne soient pas susceptibles d'arrêter le développement des connaissances réelles, puisque l'enthousiasme passager qu'elles excitent encore ne tient essentiellement qu'à des circonstances étrangères et accidentelles, il est certain qu'elles exercent une influence funeste, en retardant dans beaucoup de têtes, le développement du véritable esprit philosophique, et en consumant en pure perte une grande activité intellectuelle ${ }^{13}$.

Broussais, par contre, est l'un des trop rares esprits judicieux ayant compris et dénoncé la "vague et chimérique " psychologie des métaphysiciens, et leur «prétendue méthode d'observation intérieure ». Mais par-delà ces louanges appuyées des attaques de Broussais, Comte les trouve encore trop timides et les renforce sur quatre points ${ }^{14}$. a) Broussais " aurait pu aborder la question d'une maniere encore plus directe, en prouvant immédiatement qu'une telle observation intérieure est nécessairement impossible ". Comte développe alors l'argumentation ébauchée dix ans plus tôt ${ }^{15}$. b) S'il souscrit pleinement à la manière dont Broussais a stigmatisé les étroites limites d'une psychologie qui ne considère que \& l'homme adulte et parfaitement sain », faisant abstraction et de ses déterminations physiologiques animales et des états de développement impar-

13. S., IV, App., p. 217-218.

14. Ibid., p. $219-221$.

15. Ibid. : " L'homme ne saurait directement observer ses opérations intellectuelles, il ne peut en observer que les organes et les résultats. Sous le premier rapport, on rentre dans la physiologie; sous le second, les grands résultats de l'intelligence humaine étant les sciences, on rentre dans la philosophie des diverses sciences, qui n'est point séparable des sciences elles-mêmes. Sous aucun rapport il $n^{\prime} y$ a de place pour la psychologie ou étude directe de l'âme indépendamment de toute considération extérieure. " 
faits et des états pathologiques, Comte le trouve trop généreux envers ladite psychologie, et trop discret sur la supériorité de la physiologie et de ses approches comparatives. c) Comte reproche aussi à Broussais d'avoir encore trop épargné l'idéologie et l'ontologie, et de n'avoir pas assez souligné la rupture radicale nécessaire : celles-ci, lors même qu'elles tenaient compte de l'action des sens externes dans notre intelligence, négligeaient de renvoyer aux "prédispositions des organes cérébraux internes », que Charles Bonnet, Cabanis, Gall et Spurzheim avaient cependant soulignées. d) Enfin, Comte juge que Broussais n'a guère mieux circonscrit « le véritable champ de la physiologie » que ses prédécesseurs : comme eux, il a confondu « l'étude de l'homme individuel et celle de l'espèce humaine envisagée dans son développement collectif ». S'ils ont bien vu que ce que l'on fait généralement relever de la psychologie, relève de la physiologie, ils n'ont pas compris que certains faits et phénomènes relèvent d'une science dont il faut reconnaître l'originalité, la "physique sociale ".

Cet examen ambivalent a donc pour enjeu de redéfinir les territoires : là, Comte trouve que Broussais en donne encore trop, à l'introspection, à l'idéologie, à l'ontologie, et pas assez à la physiologie et à la philosophie des sciences; mais ici, il trouve que la physiologie devient imperialiste, qu'on ne lui donne pas ses limites, et qu'elle ne saurait empiéter ou s'annexer l'étude de l'espèce, de l'histoire et des collectifs sociaux.

Ainsi les méditations comtiennes - historiques, politiques, scientifiques, logiques, médicales - convergent : la "psychologie » traditionnelle n'est pas une science, mais il faut trouver comment étudier scientifiquement ce qu'elle prétendait connaître. Qui peut la relayer? comment en redistribuer les objets pour les rendre accessibles à une méthode positive?

4) Le Cours de philosophie positive procède à la redéfinition des champs et des frontières. Tout en proclamant le non-lieu de la psychologie, il propose des solutions de substitution. Mais pour pouvoir avancer celles-ci, il faut s'être radicalement débarrassée de celle-là : ce que font les critiques comtiennes redéployées.

L'un des textes majeurs est dans la leçon d'ouverture, ce qui traduit l'importance de la question pour Comte. C'est une synthèse nouvelle et complétée des critiques précédentes ${ }^{16}$. La psychologie est présentée comme le dernier bastion, le dernier refuge plutôt, des métaphysiciens et des théologiens. Et tout est renvoyé à l'étude physiologique comme modèle et concurrente $^{17}$. Les attaques majeures portent encore sur la méthode. Une

16. $C_{1}, 1830,1^{m}$ l., I, p. 33-34.

17. Ibid. : * On voit que, sous aucun rapport, il n'y a place pour cette psychologie illusoire, demière transformation de la théologie, qu'on tente si vainement de ranimer aujourd'hui, et qui, sans s'inquiéter ni de l'étude physiologique de nos organes intellectuels, ni de l'observation des procédés rationnels qui dirigent effectivement nos diverses recherches scientifiques, 
apparente générosité - Comte admet une observation possible des « passions » - ne fait que permettre de renforcer la critique ${ }^{18}$. Et Comte d'ajouter maintenant des commentaires ironiques et perfides sur les résultats obtenus : ils sont maigres, et toujours divergents; il y a une multitude d'écoles qui disputent sans cesse; les quelques notions véritables obtenues l'ont été en fait par d'autres moyens, ou par inadvertance : elles ne sont " dues qu'à l'infidélité des psychologues à leur prétendue méthode », et sont de toutes façons «clairsemées » et «bien inférieures aux remarques déjà faites sans ostentation par les savants sur les procédés qu'ils emploient $»^{19}$. Bref, la psychologie est décidément périmée, et la méthode la plus élémentaire et spontanée des vrais savants lui est supérieure.

En 1837 , Comte revient à la charge ${ }^{20}$. Il précise qui il vise : les « principales écoles actuelles »:

«c'est-à-dire ce qu'on nomme l'école française, l'école allemande, et enfin la moins consistante et aussi la moins absurde de toute, l'école écossaise, en tant

prétend arriver à la découverte des lois fondamentales de l'esprit humain en le contemplant en lui-même, c'est-à-dire en faisant complètement abstraction et des causes et des effets [...] Les métaphysiciens livrés à l'étude de notre intelligence n'ont pu espérer de ralentir la décadence de leur prétendue science qu'en se ravisant pour présenter les doctrines comme étant fondées aussi sur l'observation des faits. $\dot{A}$ cette fin, ils ont imaginé, deux sortes d'observations d'égale importance, l'unę extérieure, l'autre intérieure, et dont la dernière est uniquement destinée à l'étude des phénomènes intellectuels [...] On croyait, il y a encore peu de temps, avoir expliqué la vision, en disant que l'action lumineuse des corps détermine sur la rétine des tableaux représentatifs des formes et des couleurs extérieures. À cela les physiologistes ont objecté avec raison que, si c'étaient comme images qu'agissaient les impressions lumineuses, il faudrait un autre ceil pour les regarder. N'en est-il pas encore plus fortement de même dans le cas présent? Il est sensible en effet, que, par une nécessité invincible, l'esprit humain peut observer directement tous les phénomènes, excepté les siens propres. Car par qui seraient faites les observations?

18. Ibid. : « On conçoit, relativement aux phénomènes moraux, que l'homme puisse s'observer lui-méme sous le rapport des passions qui l'animent, par cette raison anatomique, que les organes qui en sont le siège sont distincts de ceux destinés aux fonctions observatrices. Encore que chacun ait eu occasion de faire sur lui de telles remarques, elles ne sauraient évidemment avoir une grande importance scientifique, et le meilleur moyen de connaître les passions sera-t-il toujours de les observer du dehors; car tout état de passion très prononcé, c'està-dire précisément celui qu'il serait le plus essentiel d'examiner, est nécessairement incompatible avec l'état d'observation. Mais quant à observer de la même manière les phénomènes inteliectuels pendant qu'ils s'exécutent, il y a impossibilité manifeste. L'individu pensant ne saurait se partager en deux, dont l'un raisonnerait, tandis que l'autre regarderait raisonner. L'organe observé et l'organe observateur étant dans ce cas identiques, comment l'observation pourrait-elle avoir lieu? Cette prétendue méthode psychologique est donc radicalement nulle dans son principe. Aussi considérons à quels procédés profondément contradictoires elle conduit immédiatement! D'un côté, on vous recommande de vous isoler autant que possible, de toute sensation extérieure, il faut surtout vous interdire tout travail intellectuel : car, si vous étiez seulement occupés à faire le calcul le plus simple, que deviendrait l'observation intérieure? D'un autré cồté, après avoir, enfin, à force de précautions, atteint cet étal parfait de sommeil intellectuel, vous devez vous occuper à contempler les opérations qui s'exécuteront dans votre esprit lorsqu'il ne s'y passera plus rien! $»$

19. C., $1^{\text {re }} 1 ., 1$, p. 33-34.

20. C., $45^{e} 1$,. I, p. $851-863$. 
du moins qu'on peut concevoir aucune véritable école dans une philosophie qui engendre autant d'opinions inconciliables qu'elle rencontre d'adeptes doués de quelque imagination. On peut d'ailleurs s'en rapporter pleinement à ces diverses sectes pour la mutuelle réfutation de leurs différences les plus profondes $»^{21}$.

Leur trait commun, du point de vue de la méthode, est « leur vain principe fondamental de l'observation intérieure » dont Comte redit la « profonde absurdité » et les contradictions. Du point de vue des doctrines, « l'aberration fondamentale essentiellement commune à toutes les sectes » est « la fausse appréciation des caractères généraux entre les facultés affectives et les facultés intellectuelles » et leur préoccupation spéculative à peu près exclusive pour " l'esprit », liée à l'affirmation d'une unité du « moi » correspondant à une unité de l'«âme ${ }^{22}$. En fait, l'«idéologie » est la métaphysique sous-jacente à toutes les sectes : Comte emploie d'ailleurs les deux mots «psychologie» et «idéologie» comme synonymes ${ }^{23}$. Comte défend aussi la substitution de l'approche phrénologique aux prétentions psychologiques et propose une "physiologie phrénologique " qu'il juge d'ailleurs « entièrement à faire " ${ }^{24}$, car on s'est jusqu'ici trop restreint à de vains essais de localisations ${ }^{25}$.

21. Ibid., p. 853; la critique de l'école française englobe aussi bien Cousin - Comte reprend ici la critique de Broussais contre « la déplorable manie psychologique qu'un fameux sophiste avait momentanément réussi à inspirer à la jeunesse française " (p. 854); que Destutt de Tracy - il aurait plutôt bien commencé, mais est rentré bientôt « dans les voies ordinaires de l'aberration métaphysique * et a « rétrogradé réellement en-deçà de l'ancienne discipline scolastique » (p. 855); que Condillac - « les rêveries puériles de Condillac et de ses successeurs sur la sensation transformée, pour « représenter les différents actes intellectuels comme finalement identiques » étaient dominées par une « vaine tendance à l'unité * et ont mené à des « conceptions fantastiques » (p. 860); et Helvétius - Comte voit une « évidente filiation » entre l'école française de l'Idéologie et Helvétius chez lequel il condamne * le double paradoxe sur l'égalité fondamentale de toutes les intelligences humaines, en tant que pourvues des mêmes sens extérieurs, et sur l'égoïsme érigé en principe nécessairement unique de toute nature morale " (p. 862). L'école allemande est accusée de défendre des doctrines d'un "vague absolu " et « inintelligibles ", qui n'ont guère de sens que par rapport à celles de l'école française dont elles sont une " imparfaite réfutation » (p. 861). L'école écossaise est bien inconsistante, sans grande liaison ni d'ailleurs influence; Comte apprécie cependant qu'elle ait insisté sur le ressort de la « sympathie ".

22. Ibid., p. 856-857.

23. Ibid., p. 855-856. La synonymie est faite tant pour la méthode (p. 855 : « la méthode psychologique ou idéologique ») que pour la doctrine (p. 855 : "la psychologie ou idéologie »). On signalera que dans le Systeme, Comte fait une distinction entre les « psychologues * et les « ldéologues » présentés même comme leurs « rivaux naturels » $(S .$, I, « Discours préliminaire », p. 73), ce qui renvoie aux éloges partiels déjà faits dans le Cours sur Destutt de Tracy, qui avait pris un bon départ en déclarant «l'idéologie est une partie de la zoologie ": l'idéologie s'inscrivait ainsi dans un souci de lier études du corps et de l'âme, ou selon les mots de cet autre Idéologue, Cabanis, d'envisager des « Rapports du physique et du moral ".

24. lbid., p. 851 note et p. 876 .

25. Comte en refuse fermement bien des thèses : il trouve la localisation proposée par Gall " effectivement hasardée et même notoirement erronée à beaucoup d'égards essentiels » 
On voit mieux alors quelle «psychologie » est ainsi répudiée comme pseudo-science, et pourquoi. Pour faire vite je dirai que c'est tout ce qu'on pourrait appeler "égologie ». C'est aussi tout ce qui prétend établir une " logique ", une connaissance des lois de l'entendement, comme base de la réflexion philosophique, à l'ancienne mode des scolastiques comme à la mode nouvelle de l'idéologie : c'est commencer par ce qui ne peut être qu'établi à partir de l'examen des sciences et de leurs performances, et prétendre maitriser les méthodes sans les lier à leur effectivité, à leur mise en œuvre dans les doctrines ${ }^{26}$.

À reprendre les critiques de Comte, on s'aperçoit aussi qu'il ne s'agit pas de renoncer aux problèmes traités, ou d'interdire ces recherches, mais de réclamer qu'ils le soient autrement.

\section{2. - LES REDISTRIBUTIONS.}

1) C'est d'abord par la biologie, par l'étude des fonctions cérébrales grâce à la «physiologie phrénologique », que Comte propose de faire l'étude positive des dispositions, des facultés intellectuelles, affectives, morales ${ }^{27}$. Comme pour tous les phénomènes organisés, la méthode principale est la comparaison ${ }^{28}$ à partir d"observations multiples obtenues sur des ensembles d'individus.

Dans le Cours, Comte s'en tient sur ces sujets à quelques propositions très génerales : "l'innéité des diverses dispositions fondamentales, soit affectives, soit intellectuelles »; « la pluralité des facultés essentiellement distinctes et radicalement indépendantes les unes des autres ${ }^{29}$; une localisation volontairement grossière ${ }^{30}$. Il tient cependant des propos doctrinaux

(ibid, p. 871), cette * distribution arbitraire et désordonnée $*$ (ibid, p. 873) et il critique « l'exorbitante multiplication des facultés fondamentales * (ibid., p. 875).

26. La nécessité de lier méthodes et doctrines est un leitmotiv de la philosophie des sciences de Comte : voir, par ex., C., $2^{e} l_{1}$, I, p. $62 ; 58^{t}$ I., H, p. 740-741; D.E.P., p. 105, etc.

27. Rappelons le titre de la $45^{*}$ leçon: :Considérations générales sur l'étude positive des fonctions intellectuelles et morales, ou cérébrales, *

28. C., $40^{e}$ I., 1, p. $699 \mathrm{sq}$.

29. $C, 45^{\mathrm{E}} 1,1, \mathrm{p}, 863$.

30. Comte trouve d'une a profonde justesse * la principale subdivision des deux ordres essentiels de facultés et d'organes, voir ibid., p. 867 ; «c'est-ă-dire la distinction des facultés affectives en penchants et sentiments ou affections, dont les premiers résident dans la partie postérieure et fondamentale de l'appareil cérébral, tandis que sa partie moyenne est essentiellement affecté aux autres; et pareillement, la distinction des facultés intellectuelles en diverses facultés perceptives proprement dites, dont l'ensemble constitue l'esprit d'observation et un petit nombre de facultés éminemment réflectives, les plus élevées de toutes, composant l"esprit de combinaison; la partie antéro-supérieure de la région frontale étant le siềge exclusif de ces dernières, principal attribut caractéristique de la nature humaine. $m$ De la phrénologie de Gall et Spurzheim, Comte, in ibid., p. 869, ne retient donc guère comme incontestable que la distinction globale entre a ce qu'on nomme le caeur, le caractère et l'esprit $*$, et, 
assez provocants : sur les liens entre animaux et hommes ${ }^{31}$; ou sur le renversement des ordres de subordination entre facultés affectives et intellectuelles $^{32}$; ou encore quand il rapporte des vertus morales à des synergies cérébrales ${ }^{33}$. La «biologie positive » de Comte a décidément transformé les fonctions « spirituelles » en « fonctions cérébrales».

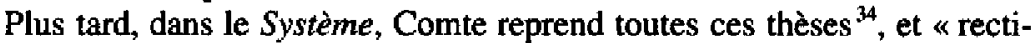
fiant » largement d'ailleurs les propos de $\mathrm{Gall}^{35}$, il élabore ce qu'il appelle "la nouvelle théorie cérébrale *. On renverra au Tableau qui répertorie dix-huit fonctions ${ }^{36}$, pour ne retenir ici que le thème martelé de la « prépondérance naturelle de l'affection sur la spéculation et l'action ${ }^{37}$, et l'importance de la division de la «vie affective » en « personnalité » et « sociabilité » pour les animaux supérieurs et a fortiori pour l'homme ${ }^{38}$.

2) Mais par la biologie on n'a affaire qu'à l'être vivant, à l'être organisé, et fût-ce par une « physiologie phrénologique » bien au point, somme toute à l'animal singulier. Pour avoir accès aux fonctions les plus complexes, et originalement humaines ${ }^{39}$, il faut une autre science positive,

comme d'ordinaire, il voit un argument de validation dans cette convergence entre théorie savante et propos de "bon sens vulgaire ", * vaguement établi de tout temps ».

31. Voir, par ex., ibid, p. 857, 859, 866, 877-878. Comte n'hésite pas à reconnaitre une * nature morale des animaux "et il va même jusqu'à affirmer que leur infériorité n'est vraiment due qu'à l'imperialisme de l'essor humain.

32. Voir, par ex., ibid., p. 860-867.

33. Voir ibid, p. 879 : « Sans doute le martyr qui supporte, avec une fermeté inébranlable, les plus horribles supplices pour éviter seulement le désaveu solennel de ses convictions, le savant qui entreprend une expérience périlleuse... etc., pourraient fuir le champ de bataille $s$ "ils étaient forcés à combattre pour une cause qui ne leur inspirerait aucun intérêt : mais leur genre de courage n'en est pas moins essentiellement identique au courage spontané et animal qui constitue la bravoure militaire proprement dite; il n'y a, entre tous ces cas d'autre différence principale que l'influence supérieure des facultés intellectuelles. "

34. Voir $S ., \mathrm{I}, \mathrm{p} .670-732$.

35. Voir $S$., I, p. 674-675: Gall a eu «l'heureuse audace de proclamer la prépondérance positive du cceur sur l'esprit $»$, mais il aurait selon Comte encore accorde une « attention trop exclusive aux nobles fonctions qu'il avait placées au sommet de l'organisme », et il ne liait pas assez ni les fonctions entre elles, ni au reste de l'organisme. Bref son génie était « trop analytique * (ibid, p. 673). Comte corrige aussi la « vaine distinction entre "penchants" et "sentiments" " (ibid., p. 680) et refusant toujours de "s'arrêter à aucune localisation spéciale *, il ne parle que d' "avant " et d' " arrière » séparés par le « niveau moyen » (ibid., p. 681-682).

36. S., 1, p. 679-680, 729 : Comte dit avoir constamment travaille cette thérie depuis 1846 ; il a fait une dizaine de tableaux avant celui du Systeme. Le tableau est présenté p. 726 bis. Il est ré́dité en 1852 dans le Catéchisme positiviste, puis en 1855 dans l'Appel aux conservateurs.

37. S., I, p. 689.

38. $S_{.}$I, p. 691. Ces thèses commandent ce que Comte appelle « le grand problème humain $n$ : subordonner autant que possible la personnalité à la sociabilité, en rapportant tout à l'Humanité », voir $S_{1}, I_{4}$ p. 692, thèses déjà exposées dans le « D,P. \#, Conclusion; on peut dire qu'elles déterminent l'aspect psychologie collective de la * sociologie ».

39. On soulignera que pour les fonctions intellectuelles, Comte parle d'une fonction générale d'expression ou de communication englobant la mimique, l'expression orale ou écrite. 
celle que Comte appelait d'abord "physique sociale » et qu'il baptise " sociologie " ${ }^{40}$. Là seulement on a vraiment affaire à l'homme, car on a affaire à des hommes en interrelations complexes et changeantes : le propre de l'homme n'est pas d'être un animal « raisonnable $»$ * raisonneur » ou « parkant » ni même vraiment « social », mais un animal qui produit et qui est un produit de la «civilisation ${ }^{41}$. Ce qui est caractéristique, déterminant dans la « sociabilité fondamentale de l'homme», dans son "irrésistible tendance sociale ", " essentiellement spontance » ${ }^{42}$, c'est que l'unité humaine en fait n'est pas a l'individu»; il faut étudier les hommes en groupes, considérer la foule d'hommes qui constitue le seul être humain vraiment réel : le «Grand-Être » qu'est l'«Humanité ${ }^{43}$. La conception des hommes en est précisée : ce sont des êtres essentiellement religieux, au sens où la " religion » en termes comtiens n'est surtout pas mysticisme théologique, mais ce qui relie et rallie les hommes en une conception bien terrestre de leurs devoirs mutuels ${ }^{44}$.

Déployée en une sociologie « statique » ou étude des structures sociales, et en une sociologie «dynamique », sorte de philosophie de l'histoire qui s'appuie sur le passé pour prévoir les directions du développement de l'avenir, la nouvelle science positive fondée par Comte récupère et explore ainsi des questions de psychologie en les traitant dans la dimension collective $^{45}$ : analyses de groupes, de structures mentales, des interactions déter-

Dans le « Tableau cérébral ", il n'y a pas de place ni pour une fonction * raisonnement », ni pour la faculté de langage que Comte reconnaît « spéciale m mais tout de même existante aussi chez tous les animaux supérieurs, voir $S$., I, p. $714,715,716$.

40. C'est Comte qui construit ce néologisme : voir son imuption dans la $47^{e}$ leçon, $C$., II, p. 88. Comte fait surtout directement usage de la « théorie cérébrale $*$ en sociologie pour la partie " statique " de cette étude : voir $C ., 50^{\mathrm{f}} \mathrm{l}$., II, p. 177.

41. Voir aussi, in $C ., 48^{e}$ 1., II, p. $145 ; 50^{*}$ 1., II, p. 193, la distinction référée à Fergusson entre «animaux sociables et animaux politiques».

42. C., $50^{*}$ l., II, p. 177 sq. et $S$., II. Pour Comte « le véritable élément sociologique * est la famille. Comte montre cependant in $C ., 50^{\circ}$ I., II, p. 180-193 la prépondérance des instincts personnels dans la vie individuelle, tandis que l'ascendant continu des instincts sympathiques se fait dans la collectivité de base, la famille, pour culminer dans la société.

43. S., I, p. 334 : * L'homme proprement dit n'existe que dans le cerveau trop abstrait de nos métaphysiciens. Il n'y a, au fond, de réel que l'humanité $* ; S$., I, p. 658 : « La vague et irrationnelle notion de l'Homme continue à servir d'unité zoologique, quoique personne n'ose contester que notre vraie nature se caractérise seulement par l'Humanité »; S., II, p. 181 : «Une société n'est pas plus décomposable en individus qu'une surface géométrique ne l'est en lignes ou une ligne en points " (c'est l'auteur qui souligne). Voir aussi in S., II, chap. 3.

44. Comte ne ménage pas ses critiques des conceptions traditionnelles de la religion, tous théologismes confondus : voir, par ex., C., $50^{\mathrm{e}} 1$., II, p. 179 ; voir aussi les propos de religion comparée dans le Catéchisme positiviste, surtout dans l'Introduction, el le $11^{\mathrm{e}}$ et dernier entretien.

45. Voir notre article « La psychologie collective de Comte à Durkheim », in Actes du colloque Psychologie des foules ef politique, Naples, 1993, Naples, Éditions Liguori, 1994, p. 169-205. 
minantes entre état social, modes de vie et développements des états de pensée ${ }^{46}$.

Mais si dans la hiérarchie comtienne des sciences, la psychologie réapparaît, avec des méthodes dûment transformées, dans les deux dernières sciences, il semble bientôt qu'elle resurgit encore ailleurs et autrement. Comme si, après avoir été expulsée, délocalisée, elle subvertissait les frontières. Comme si son non-lieu la rendait toute présente. Mieux, on peut dire que Comte lui ouvre en fait un territoire nouveau au sommet de la hiérarchie des sciences.

\section{II. - LE RETOUR DU REFOULÉ}

\section{LA SEPTIÈME SCIENCE DU SYSTEME COMTIEN.}

Après le Cours de philosophie positive, on voit Comte s'intéresser de plus en plus nettement aux phénomènes «moraux ». Si bien qu'il pense bientôt pouvoir ériger leur étude en une nouvelle science positive.

À vrai dire, l'accent est mis sur la « morale » dès les méditations sociologiques du Cours' ${ }^{47}$. Mais c'est le Discours sur l'esprit positif de $1844^{48}$ qui l'exprime avec une insistance nouvelle ${ }^{49}$. Comte développe, surtout dans la deuxième partie, l'idée d'une « dernière expansion de la philosophie naturelle » au domaine moral jusqu'ici délaissé :

« La grande crise initiale de la positivité moderne n'a essentiellement laissé en dehors du mouvement scientifique proprement dit que les théories morales et sociales, dès lors restées dans un irrationnel isolement, sous la stérile domination de l'esprit théologico-métaphysique; c'est donc à les amener aussi à l'état positif que devrait surtout consister, de nos jours, la dernière épreuve du véritable esprit philosophique ${ }^{50}$.

46. On rappellera cependant que, pour être bien une « science positive fondamentale ", la sociologie comtienne évite soigneusement ce que Comte appelle « l'empirisme dispersif », et les égarements dans des recherches concrètes et détaillées jugées prématurées. Elle ne saurait non plus être tune anthropologie/ethnologie, pas plus que la «biologie » ne saurait se confondre avec l's histoire naturelle $\$$.

47. Voir surtout la $46^{\epsilon}$ leçon. Et voir les leçons de conclusion du Cours $\left(58^{\mathrm{e}}, 59^{\mathrm{e}}\right.$ et $60^{\epsilon}$ leçons). Comte renoue ici avec les analyses de son opuscule de 1826 , les Considérations sur le pouvoir spirituel.

48. Rappelons que le D.E.P. est le «Discours préliminaire " du Traité philosophique d'astronomie populaire, et que, vu son importance, Comte s'est décidé à le publier d'abord à part.

49. Ceci est nettement souligné par Paul ARBousse-Bastine dans l'introduction de sa réédition de ce Discours, Paris, U.G.C., 1963, p. $27-28$.

50. D.E.P., p. 81. Voir aussi p. 101-103: "Désormais, toutes les speculations réelles, convenablement systématisées, concourront sans cesse à constituer, autant que possible, l'universelle prépondérance de la morale », et Comte emploie tout un vocabulaire scientifique pour traduire la positivité attendue et étendue à la morale. 
En même temps, Comte juge bon de développer, à côté de la «méthode objective ", ce qu'il appelle * la méthode subjective ${ }^{51}$. Ce déplacement d'accent a été beaucoup discuté : Littré s'en est offusqué et trouve qu'il introduit une incohérence dans la philosophie positive des sciences ${ }^{52}$. Les débats ont perduré ${ }^{33}$.

En tout cas, l'usage de la « méthode subjective » change les ambitions de Comte : il pense alors pouvoir fonder ce qu'il appelle « la vraie logique humaine », conciliant « logique de l'esprit » et a logique du cour», présentées aussi comme «logique de la raison » et " logique du sentiment », ou encore comme $«$ logique rationnelle $»$ et $\alpha$ logique morale ${ }^{54}$. Ainsi, alors que la logique était l'une des formes dénoncées de la psychologie métaphysique, elle prend dans le Système une place envahissante.

Le changement du statut de la morale s'affirme. En 1848, le Discours sur l'ensemble du positivisme ${ }^{55}$ refait une ordonnance systématique des sciences où la "morale " devient science positive à part entière. En témoigne le plan de « l'éducation systématique » que Comte dit « tracé par la loi encyclopédique ${ }^{56}$ : il programme une année par science, mais il programme sept années, la dernière étant consacrée à la «morale ${ }^{57}$. Le Système promeut, en effet, la morale comme une « septième science », ce qui est, dit Comte, le « perfectionnement définitif de [s]a hiérarchie encyclopédique ${ }^{58}$. Ce qu'il justifie selon les critères systématiques qui ont

51. Dans le Cours, il y avait déjà des textes qui, distinguant ces démarches, appelaient à cette conciliation future : voir au début de la $40^{e}$ leçon, I, p. 666, et surtout dans la $58^{e}$ leçon. Quant à ce qu'il entend par ces deux « méthodes ", Comte en fait une présentation synthétique et comparé au début de I" Introduction fondamentale" du Système, I, p. 443-453: " La méthode objective [...] procède du dehors au dedans, du monde à la vie »; "la méthode inverse ou subjective [...] va du dedans au dehors, de la vie au monde ».

52. Émile LITTRÉ y voit un dérapage qui entraîne trahison, et il refuse nettement de suivre son s maître "sur ce point: voir A.C.P.P., III' partie, surtout chap. 2, p. 527-537; André PoEY, in M. Littré et Auguste Comte, Paris, 1879, prend nettement parti pour les orientations finales de Comte.

53. Voir, par ex., Pierre Ducasse, Methode et intuition chez Auguste Comte, Paris, 1939; ID., Essai sur les origines intuitives du positivisme, Paris, 1939; Angèle KrEMER-MarierTI, L'Anihropologie positiviste d'Auguste Comte, Thèse, 1977, Publications Lille III, 1980.

54. Voir surtout $S .$, I, p. 450 sq. : la première logique est, dit Comte, guidée surtout par les " signes" plutôt artificiels; la seconde "fondée sur la connexité des émotions *; entre les deux, le lien est réalisé par une autre logique "qui procède surtout par images m.

55. Ce Discours publié en juillet 1848 devient, avec fort peu de modifications, en 1851 le " Discours préliminaire " du Système de politique positive, et occupe la plus grande partie du tome I.

56. «D.P. », p. 175 sq.

57. Dans le Programme de l'École positive rédigé en 1849, il y a un ajout du même type: dans la formation alors en trois ans - avec étude d'un couple de sciences par année - il est prévu de faire suivre le cours de sociologie de "vingt leçons sur la morale systématique »: voir Corr., t. V, "Annexes », p. 284; dans le Système, Comte se réfêre ensuite toujours à un " cours septenaire » : par ex., S., II, chap. vil, p. 432-433.

58. Voir $S$., II, chap. I, p. 55 ; chap. v, p. 265 ; chap. vII, p. 432-433. 
fonctionné pour l'établissement de tous les degrés de la hiérarchie : le domaine de la morale, ayant trait aux phénomènes humains individuels, s'occupe de phénomènes encore plus complexes, plus particuliers, plus dépendants et plus précis, que ceux dont s'occupe la sociologie; bref, il s'agit d'un « extrême prolongement de [s]a classification positive, d'après la généralité décroissante et la dépendance croissante ${ }^{59}$. Ainsi l'individu est-il de nouveau passible d'une étude propre. Le Catéchisme positiviste confirme l'ajout de la morale comme science de l'homme individuel, et le traduit dans le nouveau « Tableau de la Hiérarchie théorique des conceptions humaines $»$ refait par Comte en juillet $1852^{\text {6il }}$. La nouvelle science est même déclarée "la science par excellence " ${ }^{61}$. Dans le troisième tome du Système, Comte assume ceci comme «le principal contraste encyclopédique de [s]a construction religieuse envers [s]on élaboration philosophique », tout en refusant d'y voir une "contradiction " ${ }^{62}$.

Mais si les phénomènes humains individuels relèvent maintenant de la nouvelle science «morale», ceci oblige à repréciser l'objet de la « biologie » à laquelle le Cours les attribuait.

Les explications données par Comte sur cette correction montrent que la " biologie " n'exerce plus sur lui la même fascination que dans le Cours ${ }^{63}$. Comte la dépouille en fait de ses compétences pour l'étude de l'homme :

« Une conception trop vague de la biologie conduit à représenter l'étude de notre existence individuelle comme déjà comprise dans la théotie générale de la vitalité. [...] Quoique les biologistes proprement dits [...] aspirent toujours à connaître l'homme pour le modifier, cette double prétention ne se réalise que par exception. Car elle est directement incompatible avec l'irrationnelle négligence de ces grossiers penseurs envers les principaux caractères de la vraie nature humaine. Au fond, ils n'étudient en nous que l'animal et non l'homme, dont tous les attributs essentiels leur restent habituellement inconnus, davantage même qu'au vulgaire illettré, d'après leurs préoccupations corporelles. Nos prétendus médecins ne constituent réellement que des vétérinaires, mais plus mal élevés que ceux-ci ne le sont maintenant [...] La vraie biologie n'a

59. S., II, chap. vII, p. 435.

60. Caté., p. 94-97.

61. Caté., p. 100.

62. S., III, «Préambule général », p. 5 : « Celle-ci représenta la sociologie comme l'aboutissement universel; tandis qu'ici cette suprématie n'appartient qu'à la morale [...] Il n'existe réellement aucune contradiction entre ces deux appréciations successives, puisque ma sociologie renfermait d'abord la morale quoique confusément. La séparation normale de ces deux sciences se borne donc à perfectionner la hiérarchie abstraite, en poussant plus loin l'application de mon principe encyclopédique. "

63. Ce changement se repère aussi dans l'éloignement de Comte par rapport à Blainville : le Cours ne cessait d'en faire l'éloge; mais les relations se sont dégradées : voir le Discours sévère prononcé par Comte aux funérailles de Blainville le 7 mai 1850, publié en Annexe de S., I. 
nullement pour objet la connaissance individuelle de l'homme, mais seulement l'étude générale de la vie, envisagée surtout dans l'ensemble des êtres qui en jouissent. Elle constitue un préambule nécessaire, d'abord pour la théorie de l'ordre social, immédiatement subordonné à l'ordre vital, et enfin à la véritable appréciation de l'homme proprement dit [...] Cette conclusion normale du dogme positif se trouve aujourd'hui dépecée irrationnellement entre trois classes de penseurs, les médecins qui n'étudient que le corps, les philosophes qui croient étudier l'esprit, et les prêtres qui surtout étudient le cceur. Il résulte de ce déplorable morcellement que pas une de ces trois sortes d'intelligence ne comprend réellement la nature humaine ${ }^{64}$.

Or l'étude de la nature humaine est pour Comte l'un des propos essentiels de la science, et peut-être même le propos essentiel, puisque l'étude du monde a surtout pour souci de comprendre comment l'homme s'y insère, et s'il peut et doit le modifier. Comte adopte ainsi le terme $\alpha$ anthropologie $*$ pour désigner la science finale vers laquelle tout converge.

C'est dans le second tome dư Système que Comte fait appel à la promotion d'une «véritable anthropologie » pour la connaissance « téelle " et « véritable » de l'homme, qu'il prétend rendre enfin possible :

«Mon éminent précurseur Gall ouvrit enfin la voie qui, préparée par Cabanis et Leroy, devait aboutir à systématiser la véritable étude de l'homme, en combinant irrévocablement la connaissance positive de l'âme avec celle du corps. $\mathrm{Ni}$ les médecins, ni les prêtres n'ont encore compris suffisamment la portée d'une telle révolution scientifique. Elle n'était point appréciable avant que ma fondation de la sociologie eût terminé la préparation encyclopédique qu'exigeait l'avènement systématique de la véritable anthropologie, à laquelle il faut conserver son nom sacré de morale ${ }^{65}$.

Et Comte insiste sur l'étroite complémentarité des trois sciences : biologie, sociologie, morale-anthropologie :

«En regardant la biologie comme ébauchant l'étude de l'existence humaine, d'après celle des fonctions végétatives et animales, la sociologie fait seule connaître ensuite nos attributs intellectuels et moraux, qui ne deviennent assez appréciables que dans leur essor collectif. Dès lors, la véritable science finale, c'est-à-dire la morale, peut systématiser la connaissance spéciale de notre nature individuelle, suivant une combinaison convenable entre les deux points de vue, biologique et sociologique, qui s'y rapportent nécessairement ${ }^{66}$.

64. S., II, chap. vII, p. 437.

65. Ibid., nous soulignons. A. KREMER-MARIETTI, in op. cit. supra n. 52, a fait de l'« anthropologie positiviste », le fil conducteur de son étude du système comtien; voir aussi ID., Le Projet anthropologique d'Auguste Comte, Paris, Cedes-CDU, 1980.

66. S., II, chap. vII, p. 437-438. 
Il reste que la volonté réaffirmée de conserver à la " science finale " le nom de " morale " plutôt que d' " anthropologie " est ambiguë. À lire les textes du Système précédemment cités, on peut croire à une réserve de Comte vis-à-vis de l'anthropologie car un peu trop «biologisée », c'est-àdire «animalisée », c'est-à-dire réductrice de l'éminence proprement humaine. Ce que la «morale " respecterait mieux. Mais il faut confronter ces textes avec ceux, quasi contemporains, du Catéchisme positiviste et surtout avec le Tableau daté du 24 juillet $1852^{67}$. Ici l' " Anthropologie» est proposée pour toute l'« Étude de l'homme », en lieu et place de la «Sociologie » élargie comprenant «biologie", « sociologie proprement dite » et «morale»: «Quand le mot Anthropologie sera plus et mieux usité, il deviendra préférable pour cette destination collective, puisqu'il signifie littéralement Étude de l'homme " ${ }^{68}$.

Soucieux d'un vocabulaire précis, mais attentif aux valeurs d'usage, dans sa recherche d'appellations adéquates, Comte est amené pour les dernières sciences à renouveler des distinctions entre l'emploi d'une même appellation en un sens plus ou moins large, entre l'emploi général et l'emploi « proprement dit ». Mais cela ne rend pas aisé la compréhension exacte des compétences attribuées aux diverses sciences, ni de leurs coordinations.

\section{2. - DÉBATS ET VARIATIONS. DES DISCIPLES INDISCIPLINÉS.}

Le statut de la «psychologie » et celui des dernières sciences comtiennes ont été parmi les questions importantes autour desquelles se sont noués débats et controverses sur, et même dans, le positivisme. Non seulement les adversaires du positivisme ont été sur ces points très ardents, mais différents adeptes du positivisme, et non des moindres, en ont aussi souvent et âprement débattu : ce fut l'un des points de mésentente entre John Stuart Mill et Auguste Comte; et, alors que ce fut l'un des points que les disciples orthodoxes défendaient (le Dr Audiffrent, tout particulièrement), ce fut l'un des points sur lesquels Émile Littré appelait à corriger les positions de son «maître "; et ce fut l'un des points sur lesquels le Dictionnaire de médecine de Littré et Robin fut très violemment critiqué.

Nous ne donnerons ici que quelques éléments des discussions ${ }^{69}$.

67. Caté., p. 97 : « Hiérarchie théorique des conceptions humaines ou Tableau synthétique de l'ordre universel d'après une echelle encyclopédique à cing ou sept degrés."

68. Caté., p. 96, l'auteur souligne.

69. Nous les précisons dans notre thèse de Doctorat d'État, Paris 1, 1993, Heurs et malheurs du positivisme comtien, livre I, III ${ }^{\mathrm{e}}$ partie, chap. 7, 8, 9 . 
1) J. S. Mill a d'abord été un fervent admirateur de Conte ${ }^{70}$. Leurs différends, de plus en plus marqués, ont essentiellement trait à des questions relevant de la psychologie.

D'abord, la « logique » est primordiale pour Mill, et il ne dédaigne pas ce que les méditations de la « psychologie » même traditionnelle peuvent apporter. Ensuite, sur la psychologie même, et bien que Mill essaie de les minimiser, des désaccords apparaissent et s'amplifient très vite :

« Je dois m'avouer suspect à vos yeux de tendances métaphysiques, en tant que je crois à la possibilité d'une psychologie positive, qui ne serait certainement ni celle de Condillac, ni celle de Cousin ni mêtme celle de l'école écossaise, et que je crois toute comprise dans cette analyse de nos facultés intellectuelles et affectives, qui entre dans votre système comme destinée à servir de vérification à la physiologie phrénologique, et qui a pour but de séparer les facultés vraiment primordiales de celles qui ne sont que les conséquences nécessaires des autres ${ }^{7 !}$.

Le Système de logique, paru en 1843, répercute, quoique de la façon la plus conciliante possible, ces différends.

«C'est ce qu'on doit comprendre que M. Comte veut dire quand il réclame la connaissance scientifique des phénomènes moraux et intellectuels pour les seuls physiologistes, non seulement déniant à la psychologie ou philosophie mentale proprement dite le caractère de science, mais encore en la plaçant, quant à la nature chimérique de son objet et de ses prétentions, sur le même niveau que l'astrologie. Mais il demeure incontestable chez $\mathrm{M}$. Comte et chez les autres, qu'il existe des uniformités de succession entre les états mentaux, et que ces uniformités peuvent être constatées par l'observation et l'expérience [...] Les successions qui se passent dans nos phénomènes mentaux ne se laissent donc pas déduire des lois physiologiques de notre organisation nerveuse; et toute connaissance réelle sur leur compte doit continuer pour longtemps, sinon pour toujours, à être cherchée dans l'étude directe, par observation et expérience. Puisque l'ordre de nos phénomènes mentaux doit être étudié dans ces phénomènes et non inféré des lois de quelques phénomènes généraux, il y a une science de l'esprit distincte et séparée $"$ "

La revendication d'une « psychologie » comme science à part entière est donc maintenue par Mill. Les différends deviennent des affrontements lorsque, sur les conseils de Comte, Mill s'efforce de comprendre les rapports prévus par Comte entre la « psychologie positive » et l'analyse phré-

70. Entre 1840 et 1846 surtout : voir leurs correspondances, in Corr, t. II à IV.

71. Corr., II, lettre à Comte, 18 déc. 1841 , p. 348.

72. Système de logique, t. II, p. 499. 
nologique ${ }^{73}$; et, en particulier, lorsque Mill découvre que Comte appuie sur la biologie et sur sa théorie cérébrale certaines conceptions de la psychologie féminine et des destinées sociales des femmes qui le scandalisent ${ }^{74}$. Comte et Mill argumentent : l'un arguant surtout à partir de la biologie, l'autre surtout de l'expérience historique et sociologique. Les deux correspondants restent sur leurs positions inconciliables ${ }^{75}$.

Vingt ans après la fin de cette correspondance, Mill publie un texte, Auguste Comte and Positivism, de mises au point ${ }^{76}$. Mill se déclare farouchement opposé au comtisme des dix dernières années. S'il persiste à déclarer sa « haute admiration » pour la «quantité et la qualité de puissance mentale » de Comte ${ }^{77}$, s'il réitère son accord sur la philosophie de l'histoire des conceptions humaines, sur la « remarquable conception d'une échelle de subordination des sciences » et leur « merveilleuse systématisation ", s'il dit apprécier aussi la fondation de la sociologie, s'il défend même certains aspects des prolongements religieux du positivisme comtien $^{78}$, s'il sauve des dernières cuvres encore la promotion de la «morale " comme septième science ${ }^{79}$, et défend même ces thèses de

73. Voir les échanges entre fin février et juin 1842.

74. Voir les échanges de lettres de 1843. La question des relations hommes-femmes commence à être abordée entre les deux philosophes à partir d'une confidence de Comte sur sa séparation décisive d'avec sa femme Caroline, voir Corr., II, lettre à Mill, 24 août 1842, p. 76-77.

75. Plus tard, Comte redéveloppe ces positions, et les présente comme celles d'un véritable féminisme, car il prétend participer à la vraie et seule promotion possible de la fermme : voir S., I, «D.P. », IV" partie; voir le Catéchisme positiviste, dont « La Femme » est la catéchumène, et les développements du Système de politique positive, surtout t. II, chap. III ; voir aussi notre article "Le féminisme militant d'un auguste phallocrate ", en collab. avec Bernadette Bensaude-Vincent, Revue philosophique, 3, 1976, p. 293-311. De son côté, J. S. MrL milite contre L'Assujettissement des femmes: le texte, publié en 1869 , mais écrit huit ans auparavant, réunit et développe entre autres les arguments déjà débattus avec Comte.

76. Elles développent des idées parues en 1864 dans deux articles dans la Westminster Review. Mut évoque aussi ces relations in Mes mémoires, trad., Paris, Alcan, 1888, par ex., p. 203-204.

77. A.C.P., p. 3.

78. A.C.P., p. 135, 137, 139 : « Il ne changea rien à l'attitude purement négative qu'il gardait à l'égard de la théologie : sa religion est sans Dieu [...] Nous osons penser qu'une religion sans Dieu peut être, mème pour des chrétiens un objet de méditations instructives et profitables [...] Nous nous joignons à ce dernier [Comte] pour mépriser, comme irrationnelle et comme vile, cette conception de la nature humaine qui consiste à la tenir pour incapable de donner son amour ou de dévouer son existence à un objet qui ne peut pas offrì, en échange une éternité de jouissances personnelles. La puissance que peut acquérit sur l'esprit l'idée de l'intérêt général de la race humaine, et comme source d'émotion, et comme motif de conduite, est une chose que beaucoup ont aperçue; mais nous ne sachions pas que personne, avant M. Comte, ait senti aussi vivement que lui et aussi complètement qu'il l'a fait toute la majesté dont cette idée est susceptible [...] Nous estimons donc, non-seulement que M. Comte était fondé à entreprendre de faire aboutir l'évolution de sa philosophie à une religion, et qu'il avait réalisé les conditions essentielles d'une religion, mais encore que toutes les autres religions s'amendent d'autant plus qu'elles se rapprochent davantage dans leur résultat pratique, de celle qu'il visait à construire. »

79. Voir A.C.P., p. 188. 
Comte contre ceux qui les ont attaquées ou prétendument « corrigées ${ }^{80}$, Mill exprime aussi franchement ses dissensions ${ }^{8]}$. Les désaccords surgis dans la correspondance sont persistants, repris et renforcés, surtout la critique de $1^{4}$ appel à la phrénologie ${ }^{82}$, et ils se focalisent alors sur une question de méthode: Mill s'élève contre le fait que Comte « rejette totalement comme un procédé sans vertus l'observation psychologique ».

«Il nous faut, pense-t-il, acquérir notre connaissance de l'esprit humain, en observant les autres. Comment nous devons observer les opérations mentales d'autrui ou en interpréter les signes, sans avoir appris, par la connaissance de nous-mêmes, la signification de ces signes; c'est ce qu'il n'établit pas ${ }^{83}$.

Par ailleurs, quant à la connaissance de l'homme, Mill critique Comte sans ménagement : sa conception de la « nature humaine » tout en « penchants » et en « instincts » antagonistes; sa conception de la vie de famille et du mariage « de l'espèce la plus orthodoxe et la plus conservatrice »; ses propositions d'organisation les plus autoritaires, etc. ${ }^{84}$. Mill réclame très haut la prise en considération de l'individuel contre l'autoritaire soumission au collectif exigée par Comte. Enfin, il n'hésite pas à faire une analyse des caractéristiques psychologiques de Comte lui-même : de son esprit systématique excessif, de son idiosyncrasie orgueilleuse et entêtée, de son intransigeance qui s'accommode pourtant de critères subjectifs et arbitraires...

Ainsi, non seulement Mill se sépare de Comte sur la psychologie en réclamant pour elle un statut tout différent, mais encore ose-t-il en user pour attaquer Comte de front. Mill se fait ainsi, de plusieurs façons, contre Comte, le champion de la psychologie.

2) Littré, qui s'est toujours proclamé « disciple » de la philosophie positive et de Comte, est peu fidèle pour ce qui touche à la psychologie.

80. En particulier contre Herbert Spencer.

81. En crescendo. Dans la première partie de A.C.P., MiLl relève des « méprises ", « défectuosités ", " points vulnérables », " lacunes », " faiblesses " et « demi-vérités », et quelques " erreurs ». Il trouve parfois Comte « effrayant " (p. 77), « superficiel " (p. 81). Mais dans la seconde partie, les erreurs deviennent " trompeuses ", " mauvaises ", " nuisibles ". Comte " perd toute mesure »: "Quand son mérite vient à être reconnu et apprécié, cette infirmité intellectuelle se trouve bientôt compliquée d'une infirmité morale. Le résultat de cette position est une gigantesque confiance en soi-même, pour ne pas dire suffisance. Celle de M. Comte est colossale. Nous n'avons rien rencontré qui en approchât " (p. 132).

82. A.C.P., p. 63-67.

83. A.C.P., p. 64.

84. A.C.P., II" partie. 
Certes, il défend la liaison des fonctions «psychiques » et du cerveau ${ }^{85}$. Pour cela, on l'accusera d'ailleurs de "matérialisme » comme Robin son co-rédacteur du Dictionnaire de médecine. Mais il est, comme Mill, pour le moins réticent sur la " physiologie phrénologique ${ }^{86}:$ l'article " Phrénologie » du Dictionnaire de médecine est d'ailleurs sévère. Littré propose ce qu'il appelle «physiologie psychique », expression qu'il prend soin de justifier $^{87}$ : il veut signifier à la fois le refus des connotations «métaphysiques » du terme traditionnel « psychologie », et la volonté positive de lier l'étude des facultés intellectuelles et morales à celle de la substance nerveuse; «physiologie cérébrale » étant par ailleurs trop large, il reste la locution « physiologie psychique » que Littré identifie parfois brièvement à «psychophysiologie». Littré demande aussi qu'on lui permette le néologisme «psychisme » à la place d'esprit ${ }^{88}$.

Dans son ouvrage Auguste Comte et la philosophie positive (1863), Littré reprend l'examen de fond en comble de la position comtienne sur la psychologie. Et les résultats sont très ambivalents. Coté critique, il utilise la méthode comtienne de la philosophie positive pour invalider la phrénologie : elle n'a pas été vérifiée par les faits ni par l'expérience, elle a «succombé devant les épreuves " ${ }^{89}$. Littré croit reconnaître, dans la persistance de Comte à s'y référer en l'absence de toute preuve, les méfaits renversants de la « méthode subjective ». Cependant, il défend l'intérêt de la question, se prononce même pour des recherches sur les localisations et la pertinence d'une physiologie cérébrale qui «en est encore à ses rudiments". D'où l'importance de l'aspect programmatique de certaines conclusions : il faut une « saine théorie cérébrale »; c'est une « lacune importante et urgente»; elle doit être partie de la biologie, et c'est la partie la plus difficile et la plus compliquée $^{90}$. Littré ne s'en tient pas là. Non seulement il faut remplacer

85. Et d'ailleurs avant même qu'il ait lu et souscrit au Cours de Comte : voir dans le recueil Médecine et médecins un article de 1840, p. 340 sq.

86. À signaler cependant que l'on retrouve, dans divers articles, l'ensemble des distributions de la théorie cérébrale comtienne : voir notre article « Positivisme, biologie, médecine : A. Comte, É. Littré, Ch. Robin », Actes du Colloque Les Savants et l'épistémologie du $x I X^{e}$ siècle, Genève, mai 1993, Paris, Blanchard, 1995.

87. Voir surtout l'article sur « Quelques points de physiologie psychique $»_{,} 1860$, repris in La Science..., X, p. 307-330: 1'article procède en fait à une sorte d'histoire de la psychologie, où dialoguent entre autres Locke, Berkeley, Hume, Destutt, Bain... sur des notions comme celles du " monde extérieur, ou objet ou non-moi », du « monde intérieur, ou sujet, ou moi », d' "expérience * avec méditations sur la "formation ", la " constitution " de ces notions, le type de leur certitude. Il affirme les bornes de l'esprit humain, pour se distancier aussi franchement du "matérialisme » que du " panthéisme " et de tout « théologisme ». Enfin, il se termine par une critique radicale de la "psychologie " à la made idéologique, éclectique et cousinienne, et par un programme de «psychologie positive» basée sur les sciences, accompagné de multiples mises en garde et d'appel à vérification.

88. La Science..., p. 320.

89. A.C.P.P., p. 545 sq.

90. A.C.P.P., p. 675. 
et compléter par ce qu'il a appelé « une physiologie psychique », mais il propose en fait une nouvelle sorte de «psychologie», tout en l'entourant de précautions et de précisions :

« D'abord, il convient d'écarter une confusion qui plane sur toute la discussion relative à la psychologie et qui l'offusque. Théorie cérébrale, théorie mentale psychologique sont pris dans deux sens très différents, que l'on ne distingue jamais. Cela signifie tantôt les conditions organiques sous lesquelles se manifeste l'intelligence, tantôt les conditions formelles sous lesquelles la connaissance s'exerce. Du moment que l'on sépare l'une de l'autre ces deux significations, on aperçoit le moyen de résoudre le débat sur le lieu de la psychologie; car à la demande : où ces deux ordres doivent-ils être étudiés? il sera répondu que le premier doit l'être dans l'anatomie, dans la physiologie, dans la zoologie, dans l'évolution des âges, dans la pathologie; il appartient donc sans conteste à la biologie; mais il sera répondu aussi que le second doit être étudié dans le développement total de l'histoire et dans l'application à tous les modes de savoir; il appartient donc sans conteste à la philosophie. Ainsi, il y a deux psychologies, l'une biologique, l'autre philosophique, l'une relative à l'homme individuel, l'autre relative à l'homme collectif, l'une fournissant ce qui est nécessaire pour passer de la biologie à la sociologie, l'autre examinant l'instrument subjectif à la lumière de tout le savoir objectif. Mais ce complément de la philosophie, je ne le nomme pas psychologie, même après définition, je l'appelle théorie subjective de l'humanité; c'est qu'en effet, s'il renferme la psychologie, il renferme beaucoup plus [...] Ce que j'appelle théorie subjective de l'humanité comprend [...] la morale, l'esthétique, et la psychologie ${ }^{91}$.

Ce texte de «disciple » est en fait très subversif. Si Littré accorde beaucoup à Comte du côté de la psychologie liée à la «biologie », il accumule cependant les infractions du côté de l'autre «psychologie » proposée: réinsertion des études des «conditions formelles de la connaissance », c'est-à-dire de la « logique » ou des « méthodes » séparées des doctrines; définition d'un champ de la «philosophie » autre que celui de l'ensemble des sciences, et sous dominance de l'histoire qui chez Comte n'est que la méthode de la sociologie; contestation explicite de la morale comme septième science ${ }^{92}$, et ajout d'un complément tripartite à la classification, référé non pas à une méthode subjective, mais à une «théorie du sujet ${ }^{93}$. Et, en clair, contestation de la

91. A.C.P.P., p. 676-677, l'auteur souligne.

92. A.C.P.P., p. 677 : “Mettre la morale en septième science, à la suite de la logique et des autres, est une faute contre la méthode; car la morale n'est point, comme les six sciences, de l'ordre objectif; et d'autre part, c'est aussi une faute contre la notion philosophique maintenant réclamée, car la morale n'est qu'une portion de l'ensemble subjectif qui doit compléter la philosophie positive."

93. A.C.P.P., p. 677 : « Les théories de la morale, de l'esthétique et de la psychologie font défaut dans la philosophie positive [...] tant qu'elles ne sont pas constituess, une foule de notions vraiment philosophiques restent déclassées, sans lieu certain, sans liaison, sans ensemble. La théorie du sujet est le complément indispensable de la théorie de l'objet. » 
rigueur et de la complétude du système demeurant « un cercle non fermé ${ }^{94}$.

Littré revient sur tout ceci dans une discussion du livre de Mill ${ }^{95}$. À vrai dire, on est un peu étonné de voir Littré développer longuement une discussion du rapport à la « psychologie » qui tenait somme toute bien peu de place dans l'ouvrage de Mill en 1865. Littré défend une sorte de compromis entre Comte et Mill : «Par le mot de psychologie, on comprend tantôt les facultés cérébrales, tantôt les produits de ces facultés. S'il s'agit d'étudier les facultés, je suis avec M. Comte; s'il s'agit d'étudier les produits, je suis avec M. Mill ${ }^{96}$.

Dans ces positions ambiguës, se révèle une constante : proposer pour la «psychologie » de nouvelles exigences, lui chercher de nouveaux territoires et de nouvelles frontières, c'est aussi reconnaître sa nécessité.

Les frontières de la « psychologie » dans le positivisme ont donc été instables et discutées. Même pour Auguste Comte, on ne saurait se contenter de se référer à l'interdit sommaire bruyamment énoncé; il inaugurait en fait une redistribution des domaines et des méthodes. Entre la biologie, la sociologie, la morale, la logique, la «psychologie » éclatée cherche une nouvelle identité, et le statut de discipline scientifique. Ce que Comte et ses disciples proclament définitivement périmé, c'est la «psychologie » qui s'appuie sur l'introspection, celle qui croit à un « esprit » sans support physiologique et cérébral, celle des « métaphysiciens », des "Idéologues » et de toutes ces écoles qui disputent sur des chimères. Mais ils veulent promouvoir une étude scientifique des « fonctions » intellectuelles, affectives, des « facultés » spéculatives, actives, morales, et de leurs « résultats »; ils défendent, une psychophysiologie, fondent une psychologie collective et tentent d'en préciser les champs et les méthodes. Les sévérités de Comte ont été atténuées par les compromis des disciples. Les débats suscités ont au moins contribué à préciser les conditions et les enjeux, en un temps où la psychologie est à un moment crucial de son histoire.

Annie PETIT, Université Blaise-Pascal, Clermont II,

29, bd Gergovia, 63037 Clermont-Ferrand (juin 1993).

94. A.C.P.P., p. 677.

95. A.C.S.M., surtout p. 29-40 : si Littré convient avec Mill de tout ce qu'il y a de douteux dans la « tentative phrénologique à laquelle il [Comte] se laissa malheureusement aller », il défend la liaison de ces recherches à la biologie, critique la confusion symétrique de Mill entre psychologie et logique (ibid., p. 30), et proclame la nécessité de faire « une doctrine du sujet » qui serait une branche de l'anthropologie (ibid., p. 39).

96. A.C.S.M., p. 30. 


\section{BIBLIOGRAPHIE}

\section{Auguste Comre :}

$E . J .=$ Écrits de jeunesse, 1816-1828, rééd. par Paulo E. de Berrêdo Carneiro et Pierre Arnaud, La Haye/Paris, Mouton (« Archives positivistes »), 1970.

$C .=$ Cours de philosophie positive, 6 vol., 1830-1842, rééd. 2 tomes, présentation et notes par Michel Serres, François Dagognet, Allal Sinaceur, Jean-Paul EinTHOVEN, Paris, Hermann, 1975.

$S .=$ Système de politique positive ou Traité de sociologie, 4 vol., 1851-1854, réimpr. in CEuvres d'Auguste Comte, t. VII, VIII, IX et X, Paris, Anthropos, 1970.

"D.P. $"=~ «$ Discours préliminaire » du Système, reproduit avec très peu de modifications le Discours sur l'ensemble du positivisme publié en 1848.

Caté. = Catéchisme positiviste, 1852, Paris, Garnier-Flammarion, 1966.

Corr. $=$ Correspondance générale et Confessions, 8 tomes, Paris, Vrin (« Archives positivistes »), 1973-1990.

Les « opuscules primitifs de l'auteur sur la philosophie sociale », repris par Comte lui-même comme «Appendice général » du tome IV avec une pagination propre, sont cités ici d'après cette édition du Système $(S$.$) :$

Appréciation = Sommaire appréciation de l'ensemble du passé moderne, 1820.

Plan = Plan des travaux scientifiques nécessaires pour réorganiser la société, $1822 / 1824$.

C.S.S. = Considérations philosophiques sur les sciences et les savants, 1825.

C.P.S. = Considérations sur le pouvoir spirituel, 1826.

\section{John Stuart MiLL:}

Système de logique = System of Logic, Londres, 1843.

A.C.P. = Auguste Comte and Positivism, Londres, 1865 ; trad. par G. ClemencEAU en 1868.

L'Assujettissement des femmes = The Subjection of Women, Londres, 1869.

Émile LITTRE :

A.C.P.P. = Auguste Comte et la philosophie positive, Paris, 1863.

A.C.S.M. = Auguste Comte et Stuart Mill, Paris, 1867.

Dictionnaire de médecine..., Paris, 1855 (en collab. avec Charles Robin).

Médecine et médecins, Paris, 1871.

La Science... = La Science au point de vue philosophique, Paris, 1873 (recueil d'articles publiés à différentes dates). 